\title{
I CAL hanno sostituito la EDD in Emilia Romagna
}

\author{
Pietro Carlo Zucchelli
}

\author{
Divisione di Nefrologia e Dialisi, Regione Emilia-Romagna, \\ Azienda Ospedaliera di Bologna, Policlinico S. Orsola-Malpighi, Bologna
}

$\mathbf{I}$ 1 mio commento al dibattito sulla emodialisi domiciliare è il seguente: «Negli anni '70 l'emodialisi domiciliare rappresentò sicuramente la tecnica o meglio l'aspetto organizzativo più nuovo nel campo della emodialisi. I risultati clinici ottenuti con l'emodialisi domiciliare, eseguita dalle ore 18-19 alle ore 23 ottenne risultati spettacolari come controllo metabolico, rieducazione clinica e qualità di vita del paziente.

In Emilia Romagna fu approvata una Legge regionale, sotto il mio impulso, che cercava di spingere e di aumentare la diffusione dell'emodialisi domiciliare con una serie anche di agevolazioni organizzative per il partner, la disponibilità regionale a offrire tutto il materiale dialitico e così via.

Alla fine degli anni '70 il numero di pazienti che si poté inviare a domicilio si ridusse progressivamente fino a pochi pazienti che poi furono tutti trapiantati e oggi non vi è più un paziente in emodialisi domiciliare in tutta l'Emilia Romagna (così almeno credo). Venne pertanto sviluppato in Regione la dialisi nei Centri ad assistenza limitata (CAL) o self-service e questo rappresenta ancora oggi l'organizzazione statisticamente più importante per l'Emilia Romagna. Il 55-
$60 \%$ dei pazienti in emodialisi periodica in Emilia Romagna sono trattati nei CAL.

Quali possibilità oggi di sviluppare nuovamente l'emodialisi domiciliare?

Credo che l'aspetto più innovativo dovrebbe essere l'emodialisi notturna e giornaliera fatta con preparazione on-line del dializzato, per 3 ore circa per volta, con un sistema automatico di preparazione molto semplificato. Questo è ciò che si tende a fare o si sta provando a fare in alcuni Centri. Sono sicuro che l'emodialisi giornaliera, serale o notturna, normalizzerebbe completamente il bilancio calcio-fosforo senza l'uso di chelanti, o con un minimo apporto di questi, ridurrebbe enormemente l'anemia e quindi il consumo di eritropoietina, normalizzerebbe i valori pressori facendo praticamente scomparire l'ipotensione intra-dialisi e forse molti altri aspetti benefici.

Vi sono però molti aspetti da risolvere: 1) la necessità di avere un sistema di preparazione molto semplice che non richieda una serie di allarmi o di monitoraggi, sì da non alterare l'eventuale riposo notturno;

2) la disponibilità in qualche modo di un partner a meno che non si voglia pensare di organizzare il tutto presso un CAL, ma a questo punto vi sarebbe la necessità di infermieri, anche se in numero limitato;

3) la necessità di considerare questa emodialisi inserita nel programma di rimborso e quindi riconosciuta a tutti gli effetti ammnistrativo-finanziariogestionale;

4) ma resta il vero problema, il più importante, quello di convincere il paziente ad andare a domicilio, di non avere il controllo medico pressoché continuo, di non avere infermieri sempre disponibili. Tutto ciò è stato quello che ha fatto fallire il programma di emodialisi domiciliare, almeno in Emilia Romagna».

pzucchelli@orsola-malpighi.med.unibo.it 